\title{
Self-similar velocity profiles and mass transport of grains carried by fluid through a confined channel
}

\author{
Miles L. Morgan, ${ }^{1}$ David W. James, ${ }^{1}$ Andrew R. Barron, ${ }^{1}$ and Bjørnar Sandnes ${ }^{1}$ a) \\ Energy Safety Research Institute, Swansea University Bay Campus, Swansea, SA1 8EN, \\ $U K$
}

(Dated: 29 October 2020)

\begin{abstract}
Confined fluid-driven granular flows are present in a plethora of natural and industrial settings yet even the most fundamental of these are not completely understood. While widely-studied grain flows such as bed load and density-matched Poiseuille flow have been observed to exhibit exponential and Bingham style velocity profiles respectively, this work finds that a fluid-driven bed of non-buoyant grains filling a narrow horizontal channel - confined both from the sides and above - exhibits self-similar Gaussian velocity profiles. As the imposed flow rate is increased and the grain velocity increases, the Gaussian flow profiles penetrate deeper into the packing of the channel. Filling fractions were observed to also be self-similar and qualitatively consistent with granular theory relating to the viscous number $I$, which at a given position on the self-similar Gaussian curve is found to be generally constant regardless of imposed flow rate or velocity magnitude. An empirical description of the flow is proposed, and local velocity and filling fraction measurements were used to obtain local grain flux and accurately recover a total grain flow rate.
\end{abstract}

\section{INTRODUCTION}

Flow of granular material through confined geometries is a phenomenon found throughout the natural and industrial worlds - one which is famously used to measure time itself in the hourglass. Processes such as suffosion in sinkhole formation $^{1}$, grain flow through fault gouges ${ }^{2}$ and seepage erosion in biogenic passages and fractures ${ }^{3}$ in addition to flow in hoppers ${ }^{4}$ and even cracked pipes ${ }^{5}$ involve the confined transport of granular matter. Perhaps the most generic law describing such flow was proposed by Berverloo in 1961, concerning the gravity-driven flow of dry grains through an aperture, typical of that in a hopper or silo ${ }^{6}$. The empirical law is still the subject of new research ${ }^{7-10}$, notably with the "free-fall arch" concept being brought into question ${ }^{11}$ and the reporting that the grain flow rate is not strictly constant with respect to filling height ${ }^{12}$. Entirely self-similar density and velocity profiles have also been found in the aperture of a silo and used to accurately obtain total grain flow rate ${ }^{13}$. When submerged in liquid, the behaviour in fact strays somewhat from Beverloo's law with a considerable increase in grain flow as the silo empties and the pressure head decreases ${ }^{14}$. The dynamics of dry silo flow with a lateral orifice have also been probed - behaviour typically conforms to the Beverloo relation except at high aperture aspect ratio while the ability to flow is also dependent on silo wall thickness and aperture orientation ${ }^{15-17}$. There are however few reports of fluid-driven flow in such a scenario. This is significant as the grain flow may no longer be restricted by the thickness of the silo walls and can subsequently occur through a horizontal conduit as in certain other granular flows like bed load transport and Poiseuille flow.

Bed load transport involves a bed of non-buoyant granular material submerged in a flowing liquid. It typically concerns a thin layer of mobile grains at the top of the bed sheared by the fluid above and is often parametrised by the Shields number,

\footnotetext{
a)Electronic mail: b.sandnes@swansea.ac.uk
}

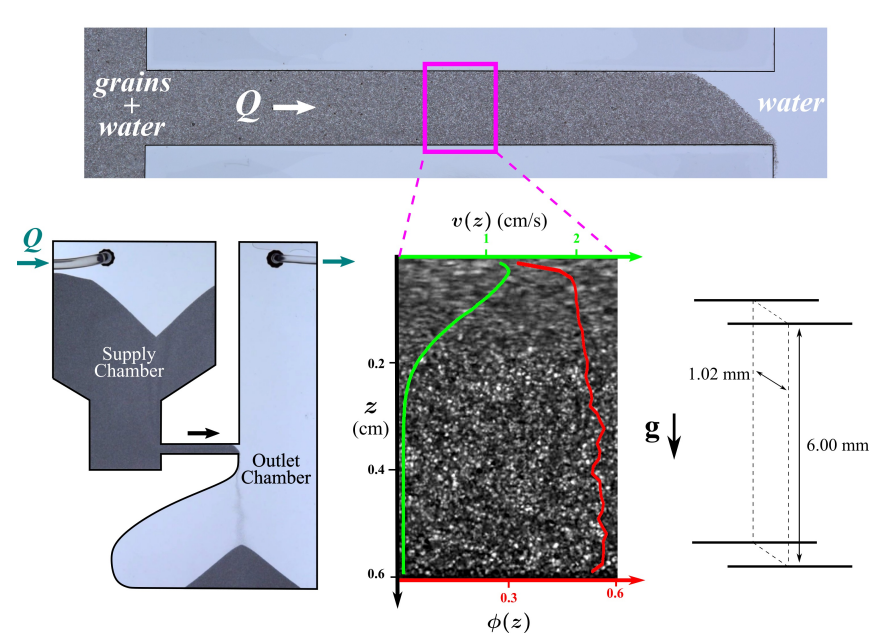

FIG. 1. The full channel (above), the whole cell (bottom left) and an enlarged image of the channel during flow at $1 \mathrm{ml} / \mathrm{min}$ (bottom middle). Velocity (green) and filling fraction (red) measurements are superimposed as a function of channel depth $z$ and a cross section of the channel is shown (bottom right). Multimedia view:

a dimensionless ratio of the fluid shear stress on the particles and gravitational stress ${ }^{18,19}$. Depth velocity profiles akin to exponential decay have been observed in the flowing region in addition to a creep flow deeper in the packing with filling fractions increasing with depth ${ }^{20,21}$ while dry grains too have been seen to exhibit similar such flow profiles ${ }^{22,23}$. Treatments of such geometries have also been carried out using theories of granular rheology including those relating to the viscous number and kinetic theory ${ }^{24-31}$. Study of Poiseuillestyle flow of grains on the other hand is somewhat limited to being either driven by gravity, for instance dry grains falling through a channel ${ }^{32,33}$, or in the complete absence of it, where pressure-driven density-matched grains in suspension exhibit Bingham-style plug flow velocity profiles similar to yield stress fluids $26,34,35$.

Here we study the dynamics of a very narrow horizontal 

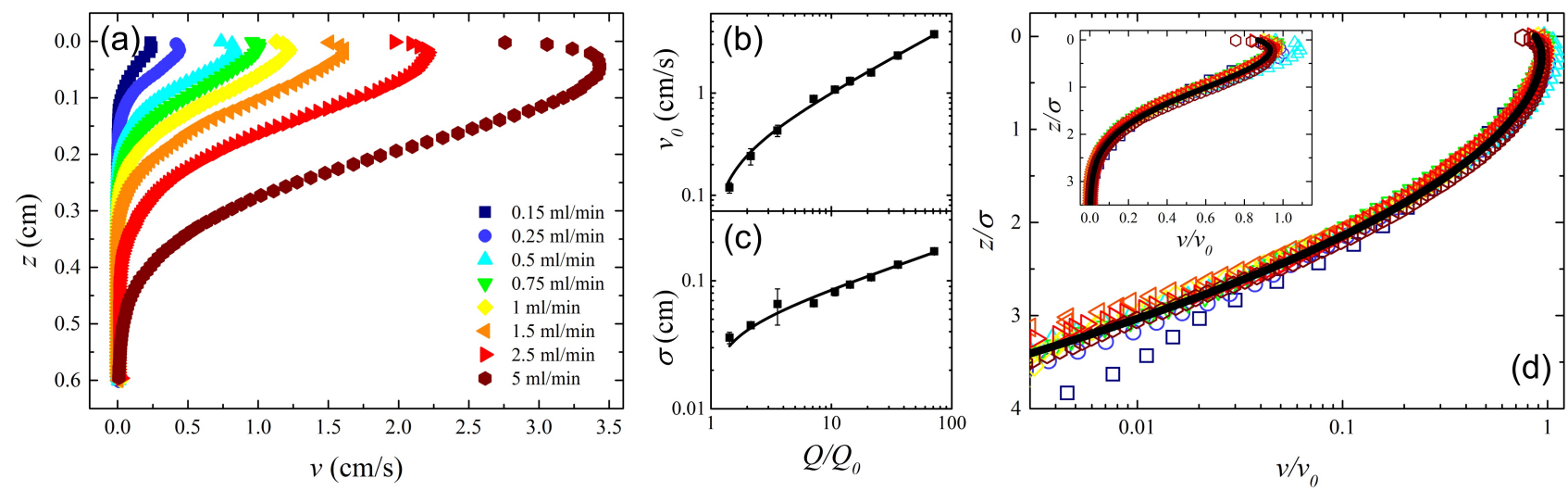

FIG. 2. (a) Grain velocity as a function of channel depth for selected imposed total flow rates. (b) Gaussian peak velocity $v_{0}$ and (c) standard deviation $\sigma$ as a function of normalised total flow rate $Q / Q_{0}$. (d) Velocity profiles normalised with $\sigma$ and $v_{0}$ fitted with Equation 2 (black line) on semi-log and (inset) linear scales.

channel filled with non-buoyant grains driven by fluid, where the granular material is confined from above and therefore always in contact with the upper boundary. Using high-speed imaging we find the grains conform to self-similar Gaussian velocity profiles with closely associated filling fractions which are described with respect to channel depth and imposed total flow rate and explored in the context of rheological theory. Finally, expressions obtained from these flow characteristics are used to successfully recover the volumetric grain flow rate as a function of total flow rate.

\section{METHODOLOGY}

The experimental flow cell comprises two large chambers connected by a straight, narrow horizontal channel. Both the chambers and the channel were laser-cut out of a sheet of polyethylene terephthalate (PET), which was subsequently sandwiched between two $20 \mathrm{~mm}$ plates of polycarbonate. This produced a $55 \mathrm{~mm}$ long flow channel of rectangular cross-section of height $h=6.00 \pm 0.05 \mathrm{~mm}$ and width $b=$ $1.02 \pm 0.02 \mathrm{~mm}$, as shown in Figure 1. Inlet and outlet holes drilled into one polycarbonate plate allow the imposition of a constant total flow rate $Q$ into the first chamber (the supply chamber), through the flow channel, and into the second chamber (the outlet chamber).

The supply chamber was filled with grains prior to the start of each experiment. After filling the apparatus with water, soda lime glass beads (sieved to between 100 and $200 \mu \mathrm{m}$ ) were introduced through the inlet and allowed to settle until the supply chamber was filled. The experiment was then initiated by imposing a flow through the system with the injection of water to the inlet by a Harvard Apparatus PHD Ultra syringe pump, causing grains to be transported through the flow channel towards the outlet chamber. Sufficient time was left before measurement to allow the flow pattern to reach a steady state. The level of grains in the supply chamber remained well above that of the flow channel throughout the experiments, and the level in the outlet chamber well below it. Therefore steady grain flow rates and velocity profiles were observed independent of packing height in the supply chamber, as in other flow rate-controlled experiments ${ }^{12}$. All measurements were carried out in triplicate.

Grain motion in the channel was captured with a Nikon 1 J4 camera fitted with a $60 \mathrm{~mm}$ macro lens at between 120 and 1200 frames per second and particle image velocimetry was subsequently performed with PIVlab software in Matlab ${ }^{36}$. Measurements were taken equidistant between the two chambers and averaged over 2 seconds of time and at least $2 \mathrm{~mm}$ of channel length to obtain depth velocity profiles $v(z)$. Due to the highly confined nature of the flow ( $b \sim 7$ grain diameters) and smooth walls there was no detectable transverse velocity gradient as would be the case in bulk flow and subsequent calculations of grain volume flux were found to be in very good agreement with independently measured volume flow rates. As such the grain velocities measured by PIV in this way are taken to be indicative of that across the whole gap.

The channel was backlit by an illuminated screen at a distance of $30 \mathrm{~cm}$. The narrow channel and translucent grains allow for estimation of the local filling fraction $\phi$ without use of index-matched media or radiation sources as seen in other work $^{25,37}$. Transmitted light intensity $J$ was mapped according to a Beer-Lambert style relationship $J=J_{0} \exp (-\varepsilon b \phi)$ where $J_{0}$ is the intensity with no grains present $(\phi=0)$ and $\varepsilon$ is a parameter related to the absorption and scattering of light, derived considering $J$ for a static bed where $\phi=\phi_{s}$.

Images of grain depletion in the supply chamber were recorded simultaneously with a second camera (Nikon 1 J2) and used to measure volumetric grain flow rate $Q_{g}$. Filling with a known volume of granular material confirmed a static grain filling fraction of $\phi_{s}=0.579 \pm 0.001$.

\section{RESULTS AND DISCUSSION}

At very low imposed total flow rate $Q$ no grain flow was observed as the interstitial fluid passed easily through the packing in a manner described by Darcy's law. However, once a 
flow threshold $Q=Q_{0}$ was surpassed, a thin layer of grains at the top of the channel became mobilised. This transition from a static state to one of fluidisation - once fluid drag had overcome granular friction - is analogous to a critical fluidisation flow rate $Q_{m}$ previously quantified by Campbell et al. with the expression $^{38}$

$$
Q_{m}=\frac{\mu \rho g k A}{\eta}
$$

where $\mu$ is the friction coefficient, $\rho$ is the effective density corrected for buoyancy, $k$ is the permeability, $A$ is the cross sectional area of the channel and $\eta$ is the fluid viscosity. Presently it is found that for $Q>Q_{0}, Q_{g}$ increases linearly with $Q$ and by extrapolation of the grain flow rate to zero flow we obtain a theoretical flow threshold $Q_{0}=0.07 \mathrm{ml} / \mathrm{min}$. While not accounting for effects due to grain polydispersity or confinement, this is close to what Equation 1 predicts as $Q_{m} \simeq 0.05 \mathrm{ml} / \mathrm{min}$ in the present conditions considering $\mu=$ $\tan \left(32^{\circ}\right)$ and $k=\frac{d^{2}}{180} \frac{\left(1-\phi_{s}\right)^{3}}{\phi_{s}^{2}}$ according to the Kozeny-Carman relation where $d$ is grain diameter, taken here to be $150 \mu \mathrm{m}$. No evidence of cohesion was observed, however for grain sizes below $d \sim 10^{-5} \mathrm{~m}$ such effects may inhibit the flow of grains and cause intermittency ${ }^{39}$. As $Q$ was further increased, grain velocity rose and the layer of mobilised grains penetrated deeper into the channel until grain flow was achieved through the full height of the packing. The results presented here consider the phase of grain flow up to the point of full channel mobilisation.

\section{Velocity Profiles}

Typical grain velocity profiles with respect to channel depth $z$ are presented in Figure 2(a) at various flow rates. In all cases a peak velocity is observed just below the upper wall with a subsequent decay deeper into the channel. Upon initial inspection a qualitative comparison may be made with flows in heaps and rotating drums which typically consider a bulk flow region of constant shear rate followed by deeper creeping region where the velocity decays exponentially ${ }^{40-43}$. However presently no clear exponential region is present and the decay is always more rapid. As such the velocities resemble more closely those of Gaussian 3D Couette flows ${ }^{44,45}$, as evidenced by the semi-log plot with depth squared $(z / h)^{2}$ in Figure 3 that shows straight lines over all measurable velocities after the peak. The sharp decrease in $v$ near the top of the channel as $z \rightarrow 0$ results from friction between the grains and the upper boundary - the continuous supply of grains ensures the channel is invariably full during flow (see Figure 1 Multimedia view). As a result, the true peak velocity is found somewhat below the top of the channel (in contrast to bed load and Couette style flows ${ }^{20,46}$ ) while the dominant Gaussian behaviour remains centred at the upper boundary $z=0$. Stress due to the cumulative weight of the grains precludes typical plug-like flow seen in other pressure or gravity driven flows between stationary boundaries ${ }^{26,33,47}$. Gaussian velocity profiles are not uncommon in granular systems and notably occur

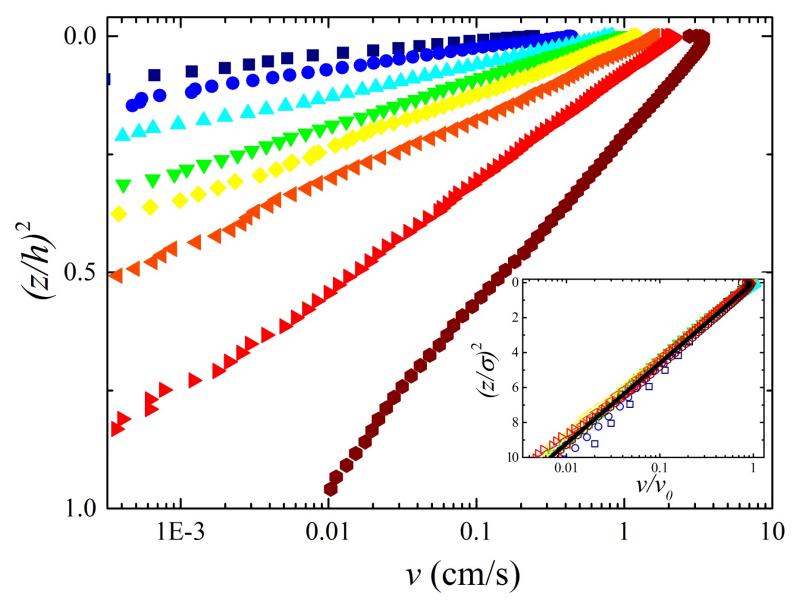

FIG. 3. Grain velocity as a function of depth squared $(z / h)^{2}$. Inset: normalised velocity $v / v_{0}$ as a function of squared normalised depth $(z / \sigma)^{2}$. Points, as in subsequent figures, represent flow rates as shown by the legend in Figure 2. The black line is normalised Equation 2.

in silo ${ }^{48-50}$ and Couette flows, as previously highlighted although they have not been observed and characterised in the context of confined fluid driven flows.

In order to build up a phenomenological description of the flow, the Gaussian amplitude $v_{0}$ and standard deviation $\sigma$ as determined by fitting are plotted as a function of normalised flow rate $Q / Q_{0}$ in Figures 2(b) and (c); error bars here and in subsequent graphs represent the standard deviation of repeat measurements. Both $v_{0}$ and $\sigma$ increase with $Q / Q_{0}$ and correspond well with power law fits with respect to $\frac{Q}{Q_{0}}-1$ (which is proportional to $Q_{g}$ ), acknowledging the presence of a flow rate threshold for grain flow such that $Q_{g}=0$ when $Q=Q_{0}$. Increases in $v_{0}$ and $\sigma$ reflect that as the total imposed flow rate is increased both the Gaussian velocity magnitude and the depth to which it penetrates the channel also increase. Normalisation of velocity profiles with respect to $v_{0}$ and $\sigma$ shows a collapse of $v(z)$ across a wide range of $Q$ in Figure 2(d) confirming their self-similar nature (only where $Q \rightarrow Q_{0}$ and the velocity approaches the lower measurable limit is there some deviation). The evolution of these velocity profiles with flow rate is therefore well-described by the modified Gaussian function fitted in Figure 2(d):

$$
v(z)=v_{0} \kappa \exp \left(-\frac{1}{2}\left(\frac{z}{\sigma}\right)^{2}\right)
$$

where $v_{0}=\alpha\left(\frac{Q}{Q_{0}}-1\right)^{0.64}$ and $\sigma=\beta\left(\frac{Q}{Q_{0}}-1\right)^{0.33}$ with $\alpha=0.24 \mathrm{~cm} / \mathrm{s}$ and $\beta=0.041 \mathrm{~cm}$ as determined by fits in Figures 2(b) and (c) (these parameters therefore represent $v_{0}$ and $\sigma$ respectively when $Q=2 Q_{0}$ ). The powers in these expressions show that as the flow rate is increased, the magnitude of the velocity profile is preferentially increased relative to the depth at which it penetrates the packing, such that approximately $v_{0} \sim \sigma^{2}$. To account for the drop in velocity at the top of the channel we include a supplementary term $\kappa$ which proposes an exponential decrease at the upper boundary such 


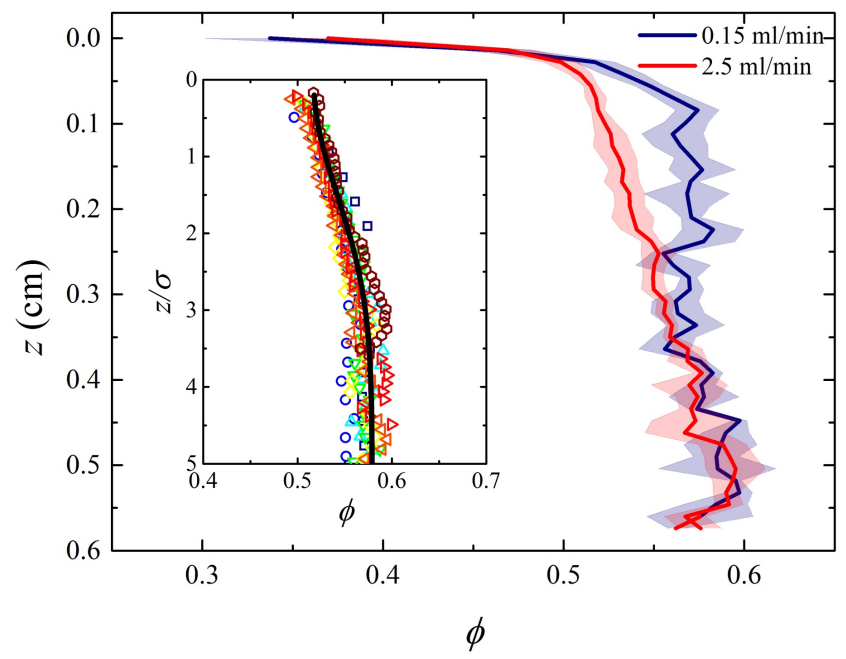

FIG. 4. Filling fraction profiles as a function of depth $z$ at typical low and high flow rates. Inset: filling fraction $\phi(z>2 d)$ with normalised depth $z / \sigma$ fitted with a modified Equation $5 \phi=\phi_{S} /\left(1+2.8 I^{1 / 2}\right)$.

that $\kappa=1-\kappa_{0} e^{-\frac{z}{0.2 \sigma}}$ where the constant $\kappa_{0} \approx 0.13$ reflects the degree of friction at the upper wall which will likely increase with grain and wall roughness. The decay length, adequately described here by $0.2 \sigma$, portrays the gradual migration of the maximum velocity deeper into the channel as the total flow rate and hence $\sigma$ are increased, with self-similarity maintained.

\section{Filling Fraction Profiles}

Filling fraction $\phi(z)$ measurements for selected flow rates are shown in Figure 4. For all $Q$, the packing is typically dense throughout the channel, exhibiting a gradual increase with depth towards the static filling fraction $\phi_{s}$. The length scale over which this increase takes place becomes larger with flow rate, highlighted by the plot of $\phi$ with normalised depth $z / \sigma$ inset in Figure 4 where a collapse of $\phi(z>2 d)$ is found across a wide range of $Q$. This self-similarity suggests association with $v(z)$ and its characteristic length scale $\sigma$. A reduction in $\phi$ is observed close to the upper wall $(z<2 d)$ which is a result of edge effects at the upper boundary. Measurements of $\phi(z)$ further illustrate disparity between the present flow and those in unconfined geometries such as bed load and heap flows, notably regarding the density throughout the packing. The faster flowing regions of other such flows are typically dilute and tend to zero at the free surface ${ }^{21,42,51}$ while those shown inset in Figure 4 demonstrate that $\phi(z) \gtrsim 0.5$, neglecting boundary effects, even at the highest velocities. Such filling fractions correspond to dense granular flow that - in free surface flows - is often observed deep in the packing accompanying slow exponentially decaying velocities, sometimes referred to as a static or quasistatic region $21,37,51,52$. As with the velocity curves of Figure 2, filling fraction similarities can in fact be better drawn between the present work and those of granular Couette flows, which are dense at the sheared surface

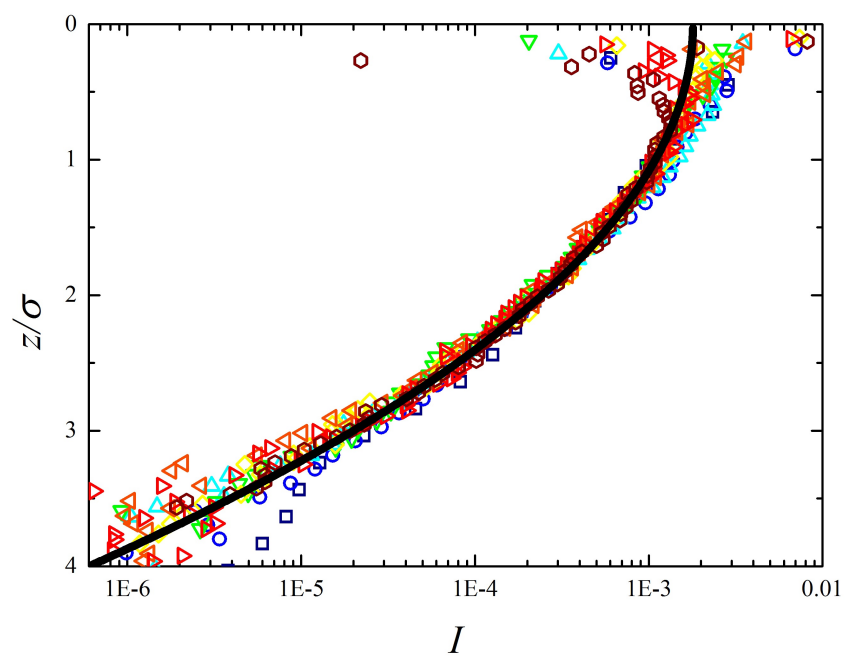

FIG. 5. Viscous number $I$ as a function of normalised depth $z / \sigma$ fitted with Equation 4 with the approximation $v_{0} / \sigma^{2} \approx \alpha / \beta^{2}$.

and further increase gradually away from it ${ }^{45,53}$. Indeed, certain aspects of commonality exist between these flows, most notably that the direction of motion is parallel to the two confining boundaries. Although the upper boundary is stationary in the present experiment, resulting in a local velocity reduction due to wall friction, the Gaussian flow is still centred upon it, as it is in Couette flows. Furthermore, preliminary bed load style experiments carried out by the authors in channels of similar dimensions to those described herein (but where the grains do not touch a confining upper wall) exhibited exponential velocity decays, and suggest that the dense granular contact with the upper boundary may be a contributor to the apparent Gaussian behaviour.

\section{Local Granular Rheology}

In order to further understand the nature of the flow and the apparent relation between the self-similar velocities and filling fractions we may consider granular rheology relating to the viscous number $I$, the ratio between micro and macro time scales of the system ${ }^{54,55}$. It is thought to be the underlying parameter in dense granular flows and has been recently assessed for beds under non-homogeneous shear ${ }^{51}$. It can be written in the form

$$
I=\frac{\eta_{f} \dot{\gamma}}{P_{p}}
$$

where $\eta_{f}$ is the interstitial fluid viscosity, $\dot{\gamma}=\frac{d v}{d z}$ is the shear rate and $P_{p}$ is the confining pressure taken to be $P_{p}=$ $\rho g \int_{0}^{z} \phi(z) d z^{20}$. Figure 5 shows $I$ calculated numerically from velocity and filling fraction measurements as a function of normalised depth $z / \sigma$, where a data collapse is achieved across most of the channel. While the self-similarity of $v(z)$ has already been demonstrated with respect to $z / \sigma$, interestingly the magnitude of $I$ is found to readily collapse. We may 


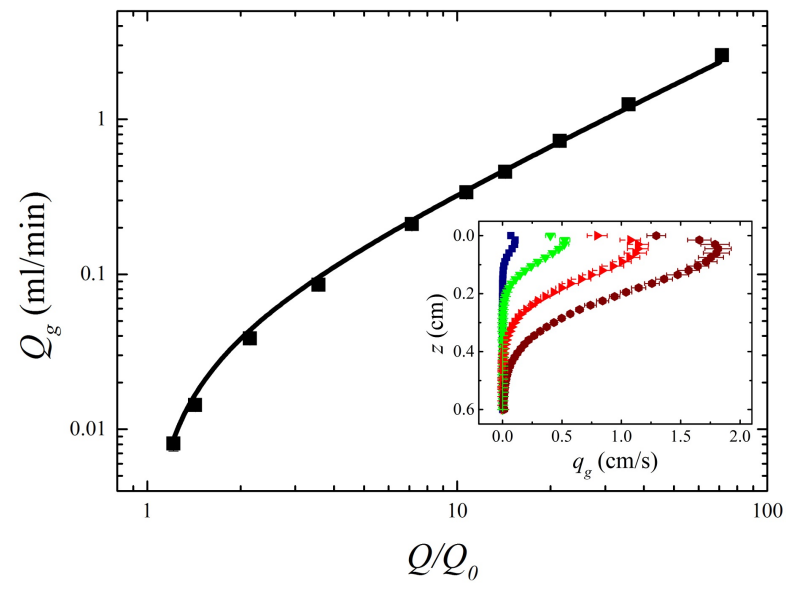

FIG. 6. Grain flow rate $Q_{g}$ as a function of normalised total flow rate $Q / Q_{0}$. Points represent experimental measurements (error bars are smaller than the data points) and the line corresponds to the numerical integration of Equation 6. Inset: local grain flux profiles calculated from measured $v(z)$ and $\phi(z)$ for selected flow rates.

explore this generally using the velocity profile of Equation 2 in conjunction with Equation 3 with some simplifying assumptions: neglecting the non-Gaussian behaviour near the upper wall $(\kappa=1)$ and approximating the confining pressure as $P_{p}=\rho g \bar{\phi} z$, where $\bar{\phi}=0.54$ represents an average filling fraction (typically $0.5<\phi(z)<0.579$ ). It therefore follows that the viscous number corresponds approximately to a Gaussian:

$$
I=\frac{v_{0} \eta_{f}}{\sigma^{2} \rho g \bar{\phi}} \exp \left(-\frac{1}{2}\left(\frac{z}{\sigma}\right)^{2}\right)
$$

As such the data collapse of Figure 5 reflects the observed evolution of the velocity profiles with $Q$ such that approximately $v_{0} \sim \sigma^{2}$ and shows that at any point $z / \sigma$ on the self-similar velocity curve, away from the upper wall, the ratio of the micro and macro time scales is equivalent regardless of flow rate or grain velocity. Equation 4 is fitted to Figure 5 with the approximation $v_{0} / \sigma^{2} \approx \alpha / \beta^{2}$. Divergence of $I$ calculated from experimental data at the upper wall is reflective of the limitations of this granular theory to account for energy flux near the boundaries.

Theory regarding the viscous number also allows for the calculation of filling fraction, proposed by Boyer et al. ${ }^{55}$ according to

$$
\phi=\frac{\phi_{s}}{\left(1+I^{1 / 2}\right)}
$$

Employing Equation 5 in conjunction with Equation 4 finds good agreement with the length scale of the increase in $\phi$ with $z / \sigma$ however somewhat over predicts $\phi$. This may be due to unconsidered confinement effects caused by the walls of the channel, resulting in the apparent $I$ being smaller than that in actuality. The inclusion of a coefficient such that $\phi=\phi_{s} /\left(1+2.8 I^{1 / 2}\right)$ provides the necessary scaling for alignment with the experimental data and is fitted inset in Figure
4. This therefore highlights the close relation between $v(z)$ and $\phi(z)$ while showing qualitative agreement with theory. It should be noted however that such analysis with respect to $I$ for velocity profiles where $\kappa<1$ (as seen in Figure 2) incorrectly predicts a maximum $\phi$ at the velocity peak where $\dot{\gamma}=0$ which is not found in experimental observation.

\section{Grain Flux}

With measurements of both local filling fraction and grain velocity, a volumetric flux profile through the channel can be obtained $q_{g}(z)=\phi(z) v(z)$. This is calculated numerically and shown for selected flow rates inset in Figure 6. A global grain flow rate $Q_{g}$ may be calculated considering laws of conservation and assuming steady, uniform flow through the channel:

$$
Q_{g}=b \int_{0}^{h} \phi(z) v(z) d z
$$

The integral of Equation 6 computed numerically using Equation 2 and the modified Equation 5 (shown inset in Figure 4) is plotted as a function of normalised flow rate in Figure 6. It is shown to be in good agreement with the data measured from the depletion of the supply chamber and hence a unification of micro-scale observations of grain dynamics and macro-scale measurements of volumetric granular flow has been achieved.

\section{CONCLUSIONS}

In summary, the behaviour of a fundamental yet unreported confined granular flow geometry has been examined. Fluiddriven flow of non-buoyant grains filling a narrow horizontal channel exhibited self-similar Gaussian velocity profiles, the peaks and widths of which increased in power-law relationships with respect to normalised imposed flow rate. Filling fractions during flow were seen to be largely self-similar and their characteristics and evolution with flow rate were linked to the Gaussian velocities through theory of granular rheology. While generally consistent with theory, the origin of the Gaussian shape is as yet not known although comparisons were drawn to Couette flows which are similarly dense and confined. Finally, velocity and filling fraction measurements were used to calculate a local mass flux profile and empirical expressions of the respective quantities enabled the accurate recovery of the total grain volume flow as a function of imposed flow rate over two orders of magnitude, reconciling micro and macro-scale measurements.

The new insights presented here may help in the understanding of physical processes in nature and industry including the formation of sinkholes, grain flow through fractures and in submerged silos. Opportunities for further study exist in the more comprehensive application of granular theory and for the investigation into the origins of the Gaussian profile in addition to the influence of size and physical properties of the grains and channel. Other theories may prove useful in understanding the present flows, such as nonlocal rheologies or kinetic theory ${ }^{27,56-60}$. Future work may consider the modelling 
of dense Poiseuille-type flow of non-buoyant grains. Furthermore the construction of the quasi-2D flow geometry invites customisation with potential avenues to the study of additional complex flow configurations.

\section{ACKNOWLEDGMENTS}

We thank James Campbell, Deren Ozturk, Dawang Zhang and Martin Monloubou for their discussions. This work was supported by the Engineering and Physical Sciences Research Council EPSRC grant EP/S034587/1 and Sêr Cymru National Research Network in Advanced Engineering and Materials Grant No. NRN141.

\section{DATA AVAILABILITY STATEMENT}

The data that support the findings of this study are available from the corresponding author upon reasonable request.

${ }^{1}$ F. Gutiérrez, J. Guerrero, and P. Lucha, "A genetic classification of sinkholes illustrated from evaporite paleokarst exposures in Spain," Environmental Geology 53, 993-1006 (2008).

${ }^{2}$ O. Dorostkar, R. A. Guyer, P. A. Johnson, C. Marone, and J. Carmeliet, "On the micromechanics of slip events in sheared, fluid-saturated fault gouge," Geophysical Research Letters 44, 6101-6108 (2017).

${ }^{3}$ T. Dunne, "Chapter 1. Hydrology mechanics, and geomorphic implications of erosion by subsurface flow," in Special Paper of the Geological Society of America, Vol. 252 (1990) pp. 1-28.

${ }^{4}$ R. Nedderman, U. Tüzün, S. Savage, and G. Houlsby, "The flow of granular materials-I," Chemical Engineering Science 37, 1597-1609 (1982).

${ }^{5}$ S. Guo and D. Z. Zhu, "Soil and Groundwater Erosion Rates into a Sewer Pipe Crack," Journal of Hydraulic Engineering 143, 06017008 (2017).

${ }^{6}$ W. Beverloo, H. Leniger, and J. van de Velde, "The flow of granular solids through orifices," Chemical Engineering Science 15, 260-269 (1961).

${ }^{7}$ C. Mankoc, A. Janda, R. Arévalo, J. M. Pastor, I. Zuriguel, A. Garcimartín, and D. Maza, "The flow rate of granular materials through an orifice," Granular Matter 9, 407-414 (2007).

${ }^{8}$ S. Dunatunga and K. Kamrin, "Continuum modelling and simulation of granular flows through their many phases," Journal of Fluid Mechanics 779, 483-513 (2015).

${ }^{9}$ J. Wan, F. Wang, G. Yang, S. Zhang, M. Wang, P. Lin, and L. Yang, "The influence of orifice shape on the flow rate: A DEM and experimental research in 3D hopper granular flows," Powder Technology 335, 147-155 (2018).

${ }^{10}$ A. M. Cervantes-Álvarez, S. Hidalgo-Caballero, and F. Pacheco-Vázquez, "The simultaneous discharge of liquid and grains from a silo," Physics of Fluids 30, 043302 (2018).

${ }^{11}$ S. M. Rubio-Largo, A. Janda, D. Maza, I. Zuriguel, and R. C. Hidalgo, "Disentangling the Free-Fall Arch Paradox in Silo Discharge," Physical Review Letters 114, 238002 (2015).

${ }^{12}$ J. Koivisto and D. J. Durian, "The sands of time run faster near the end," Nature Communications 8, 15551 (2017).

${ }^{13}$ A. Janda, I. Zuriguel, and D. Maza, "Flow Rate of Particles through Apertures Obtained from Self-Similar Density and Velocity Profiles," Physical Review Letters 108, 248001 (2012).

${ }^{14}$ T. J. Wilson, C. R. Pfeifer, N. Mesyngier, and D. J. Durian, "Granular discharge rate for submerged hoppers," Papers in Physics 6, 1-7 (2014).

${ }^{15}$ H. G. Sheldon and D. J. Durian, "Granular discharge and clogging for tilted hoppers," Granular Matter 12, 579-585 (2010).

${ }^{16}$ A. Medina, D. Cabrera, A. López-Villa, and M. Pliego, "Discharge rates of dry granular material from bins with lateral exit holes," Powder Technology 253, 270-275 (2014).

${ }^{17}$ Y. Zhou, P.-Y. Lagrée, S. Popinet, P. Ruyer, and P. Aussillous, "Experiments on, and discrete and continuum simulations of, the discharge of granular media from silos with a lateral orifice," Journal of Fluid Mechanics 829, 459-485 (2017).

${ }^{18}$ M. Ouriemi, P. Aussillous, M. Medale, Y. Peysson, and É. Guazzelli, "Determination of the critical Shields number for particle erosion in laminar flow," Physics of Fluids 19, 061706 (2007).

${ }^{19}$ S. Z. Ali and S. Dey, "Hydrodynamics of sediment threshold," Physics of Fluids 28, 075103 (2016)

${ }^{20}$ M. Houssais, C. P. Ortiz, D. J. Durian, and D. J. Jerolmack, "Onset of sediment transport is a continuous transition driven by fluid shear and granular creep," Nature Communications 6, 1-8 (2015).

${ }^{21}$ B. Allen and A. Kudrolli, "Depth resolved granular transport driven by shearing fluid flow," Physical Review Fluids 2, 1-13 (2017).

${ }^{22}$ P. A. Lemieux and D. J. Durian, "From avalanches to fluid flow: a continuous picture of grain dynamics down a heap," Physical Review Letters 85, 4273-4276 (2000).

${ }^{23}$ I. S. Aranson and L. S. Tsimring, "Continuum theory of partially fluidized granular flows," Physical Review E - Statistical Physics, Plasmas, Fluids, and Related Interdisciplinary Topics 65, 061303 (2002).

${ }^{24}$ B. Lecampion and D. I. Garagash, "Confined flow of suspensions modelled by a frictional rheology," Journal of Fluid Mechanics 759, 197-235 (2014).

${ }^{25}$ P. Aussillous, J. Chauchat, M. Pailha, M. Médale, and É. Guazzelli, "Investigation of the mobile granular layer in bedload transport by laminar shearing flows," Journal of Fluid Mechanics 736, 594-615 (2013).

${ }^{26} \mathrm{M}$. Tankeo, P. Richard, and É. Canot, "Analytical solution of the $\mu(\mathrm{I})$ - Rheology for fully developed granular flows in simple configurations," Granular Matter 15, 881-891 (2013).

${ }^{27}$ D. Berzi, J. T. Jenkins, and P. Richard, "Extended kinetic theory for granular flow over and within an inclined erodible bed," Journal of Fluid Mechanics 885, A27 (2020).

${ }^{28}$ F. Qi, S. K. de Richter, M. Jenny, and B. Peters, "DEM simulation of dense granular flows in a vane shear cell: Kinematics and rheological laws," Powder Technology 366, 722-735 (2020).

${ }^{29}$ T. Pähtz, O. Durán, D. N. de Klerk, I. Govender, and M. Trulsson, "Local Rheology Relation with Variable Yield Stress Ratio across Dry, Wet, Dense, and Dilute Granular Flows," Physical Review Letters 123, 048001 (2019).

${ }^{30}$ Y. Duan, Z.-G. Feng, E. E. Michaelides, and S. Mao, "Modified kinetic theory applied to the shear flows of granular materials," Physics of Fluids 29, 043302 (2017).

${ }^{31}$ S. Kim and K. Kamrin, "Power-Law Scaling in Granular Rheology across Flow Geometries," Physical Review Letters 125, 088002 (2020).

${ }^{32}$ K. C. Vijayakumar and M. Alam, "Velocity distribution and the effect of wall roughness in granular Poiseuille flow," Physical Review E 75, 051306 (2007).

${ }^{33}$ M. Alam and V. K. Chikkadi, "Velocity distribution function and correlations in a granular Poiseuille flow," Journal of Fluid Mechanics 653, 175219 (2010).

${ }^{34}$ T. Ahnert, A. Münch, B. Niethammer, and B. Wagner, "Stability of concentrated suspensions under Couette and Poiseuille flow," Journal of Engineering Mathematics 111, 51-77 (2018).

${ }^{35}$ D. Monsorno, C. Varsakelis, and M. V. Papalexandris, "Poiseuille flow of dense non-colloidal suspensions: The role of intergranular and nonlocal stresses in particle migration," Journal of Non-Newtonian Fluid Mechanics 247, 229-238 (2017).

${ }^{36} \mathrm{~W}$. Thielicke and E. J. Stamhuis, "PIVlab - Towards User-friendly, Affordable and Accurate Digital Particle Image Velocimetry in MATLAB," Journal of Open Research Software 2 (2014).

${ }^{37}$ P. Richard, A. Valance, J.-F. Metayer, J. Crassous, M. Louge, and R. Delannay, "Rheology of confined granular flows," in AIP Conference Proceedings, Vol. 1227 (2010) pp. 79-88.

${ }^{38}$ J. M. Campbell, D. Ozturk, and B. Sandnes, "Gas-Driven Fracturing of Saturated Granular Media," Physical Review Applied 8, 064029 (2017).

${ }^{39}$ A. Castellanos, J. M. Valverde, A. T. Pérez, A. Ramos, and P. K. Watson, "Flow Regimes in Fine Cohesive Powders," Physical Review Letters 82, 1156-1159 (1999).

${ }^{40}$ T. S. Komatsu, S. Inagaki, N. Nakagawa, and S. Nasuno, "Creep Motion in a Granular Pile Exhibiting Steady Surface Flow," Physical Review Letters 86, 1757-1760 (2001).

${ }^{41}$ B. A. Socie, P. Umbanhowar, R. M. Lueptow, N. Jain, and J. M. Ottino, "Creeping motion in granular flow," Physical Review E 71, 031304 (2005). 
${ }^{42}$ P. Richard, A. Valance, J.-F. Métayer, P. Sanchez, J. Crassous, M. Louge, and R. Delannay, Physical Review Letters 101, 248002 (2008).

${ }^{43}$ P. Richard, R. Artoni, A. Valance, and R. Delannay, "Influence of lateral confinement on granular flows: comparison between shear-driven and gravity-driven flows," Granular Matter 22, 81 (2020).

${ }^{44}$ D. M. Mueth, G. F. Debregeas, G. S. Karczmar, P. J. Eng, S. R. Nagel, and H. M. Jaeger, "Signatures of granular microstructure in dense shear flows," Nature 406, 385-389 (2000).

${ }^{45}$ D. M. Mueth, "Measurements of particle dynamics in slow, dense granular Couette flow," Physical Review E 67, 011304 (2003).

${ }^{46}$ N. Huang and D. Bonn, "Viscosity of a dense suspension in Couette flow," Journal of Fluid Mechanics 590, 497-507 (2007).

${ }^{47}$ G. D. R. Midi, "On dense granular flows,” The European Physical Journal E 14, 341-365 (2004).

${ }^{48}$ J. Choi, A. Kudrolli, and M. Z. Bazant, "Velocity profile of granular flows inside silos and hoppers," Journal of Physics: Condensed Matter 17, S2533-S2548 (2005).

${ }^{49}$ I. Zuriguel, D. Maza, A. Janda, R. C. Hidalgo, and A. Garcimartín, "Velocity fluctuations inside two and three dimensional silos," Granular Matter 21, 47 (2019).

${ }^{50}$ R. Maiti, G. Das, and P. K. Das, "Granular drainage from a quasi-2D rectangular silo through two orifices symmetrically and asymmetrically placed at the bottom," Physics of Fluids 29 (2017).

${ }^{51}$ M. Houssais, C. P. Ortiz, D. J. Durian, and D. J. Jerolmack, "Rheology of sediment transported by a laminar flow," Physical Review E 94 (2016).
${ }^{52}$ D. Bonamy, F. Daviaud, and L. Laurent, "Experimental study of granular surface flows via a fast camera: A continuous description," Physics of Fluids 14, 1666-1673 (2002).

${ }^{53}$ G. Ovarlez, F. Bertrand, and S. Rodts, "Local determination of the constitutive law of a dense suspension of noncolloidal particles through magnetic resonance imaging," Journal of Rheology 50, 259-292 (2006).

${ }^{54}$ C. Cassar, M. Nicolas, and O. Pouliquen, "Submarine granular flows down inclined planes," Physics of Fluids 17, 103301 (2005).

${ }^{55}$ F. Boyer, É. Guazzelli, and O. Pouliquen, "Unifying suspension and granular rheology," Physical Review Letters 107, 1-5 (2011).

${ }^{56}$ D. L. Henann and K. Kamrin, "A predictive, size-dependent continuum model for dense granular flows," Proceedings of the National Academy of Sciences 110, 6730-6735 (2013).

${ }^{57}$ M. Bouzid, M. Trulsson, P. Claudin, E. Clément, and B. Andreotti, "Nonlocal Rheology of Granular Flows across Yield Conditions," Physical Review Letters 111, 238301 (2013).

${ }^{58}$ V. Kumaran, "Kinetic theory for sheared granular flows," Comptes Rendus Physique 16, 51-61 (2015).

${ }^{59} \mathrm{O}$. Pouliquen and Y. Forterre, "A non-local rheology for dense granular flows," Philosophical Transactions of the Royal Society A: Mathematical, Physical and Engineering Sciences 367, 5091-5107 (2009).

${ }^{60}$ J. Goyon, A. Colin, and L. Bocquet, "How does a soft glassy material flow: finite size effects, non local rheology, and flow cooperativity," Soft Matter 6, 2668 (2010). 